\title{
Thermal expansion within a chain of magnetic colloidal particles
}

\author{
D. Lacoste,${ }^{1}$ C. Brangbour,${ }^{2}$ J. Bibette ${ }^{2}$ and J. Baudry ${ }^{2}$ \\ ${ }^{1}$ Physico-Chimie Théorique, UMR 7083 CNRS-ESPCI, \\ 10 rue Vauquelin, 75231 Paris Cedex 05, France \\ ${ }^{2}$ Laboratoire de Collö̈des et matériaux divisés, ESPCI ParisTech, \\ UPMC, UMR 7612 CNRS, 10 rue Vauquelin, 75005 Paris, France
}

(Dated: October 31, 2018)

\begin{abstract}
We study the thermal expansion of chains formed by self-assembly of magnetic colloidal particles in a magnetic field. Using video-microscopy, complete positional data of all the particles of the chains is obtained. By changing the ionic strength of the solution and the applied magnetic field, the interaction potential can be tuned. We analyze the thermal expansion of the chain using a simple model of a one dimensional anharmonic crystal of finite size.
\end{abstract}

PACS numbers: 82.70.-y,65.40.De,47.57.J-,75.50.Mm

It is well-known that the expansion of a solid with temperature is associated with the anharmonicity of the interaction between the particles of the solid. This was shown with standard solids like lead, using X-ray absorption measurements [1], and in this reference, the thermal expansion is analyzed using a one-dimensional anharmonic oscillator model. This suggests that it would be valuable to carry out such a study with a one-dimensional crystal. Although no true long range order can exist in one-dimensional systems in the thermodynamic limit, many systems are one-dimensional phases of finite length. A classical example is the mercury salts [2], but more recently, carbon nanotubes, phases of gases adsorbed inside carbon nanotubes [3], or the B phase of DNA have attracted considerable interest due to their one dimensional character.

In these one-dimensional crystals, only few experiments have investigated the thermal expansion. One reason for that is that these systems do not allow for an easy control of the interaction between th e particles (or the temperature). With colloidal systems, and in particular magnetic colloids, it is easy to obtain low dimensional condensed phases in which the interaction between the particles can be tuned. This strategy was already used in [4], where magnetic colloids were used in a beautiful experimental investigation of the 2D melting transition. To our knowledge, no comparable experimental study of 1D colloidal crystals has been carried out.

In the present letter, video microscopy is used to study the thermal expansion in a chain of magnetic colloids. At high magnetic field, such a chain can be viewed as a quasi-one-dimensional condensed phase of colloidal particles. In this system, the interaction potential can be determined experimentally from the dependence of the average interparticle distance with the applied magnetic field [5, 6]. We analyze quantitatively these experiments using Monte-Carlo simulations. As in standard solids, we find that the expansion as function of temperature is controlled by the anharmonicity of the interaction potential. In magnetic colloids, the temperature is measured by the inverse of a dimensionless number, $\lambda$ which measures the strength of the dipolar interaction with respect to the thermal energy. The anharmonicity of the interaction potential is varied experimentally by changing the ionic strength of the solution, which controls the electrostatic repulsive part of the potential.

In this paper, we describe a colloidal chain as a $1 \mathrm{D}$ lattice of $N$ colloidal particles of diameter $d$ with a nearestneighbor interaction potential $u(r)$, where $r$ is the spacing between two neighboring particles in the chain. Let us recall some well-known general features of this system before coming to the specific case of magnetic colloids. Calculations are conveniently carried out in the ensemble where the temperature $T$ and the volume $L$ (which corresponds to the length of the chain in 1D) are constant. The pressure $P$ (which corresponds to a force in 1D) is the Lagrange multiplier associated with the constraint of constant volume. Thanks to the nearest-neighbors assumption, the partition function of this problem $Z$ can be calculated exactly [7]. It is $Z=\xi^{N+1} / \Lambda^{N}$, where $\beta=1 / k_{B} T$,

$$
\xi=\int d r e^{-\beta(u(r)+P r)},
$$

and $\Lambda$ is the de Broglie length. Thus, the enthalpy $G$ equals $N k_{B} T \log (\Lambda / \xi)$ (neglecting $1 / N$ corrections). At low temperatures, this system behaves as a solid, which suggests to expand the potential $u(r)$ about its minimum obtained at $r_{\min }$. When the function $v\left(r, r_{\text {min }}\right)$ defined as $u(r)-u\left(r_{\min }\right)$, is symmetric with respect to $r_{\min }$ (by that we mean precisely $\left.v\left(r, r_{\text {min }}\right)=v\left(2 r_{\text {min }}-r, r_{\text {min }}\right)\right)$, we find that there is no thermal expansion $\langle r\rangle \simeq r_{\text {min }}$. At finite temperature, no such symmetry is present: the average of the position $\langle r\rangle$ differs from the most probable value of the position $r_{\min }$, which leads in general to thermal expansion. This can be seen using a Taylor expansion of the form

$$
v\left(r, r_{\text {min }}\right)=\frac{1}{2} k x^{2}+g x^{3},
$$

limited to cubic order with $x=r-r_{\min }$. After calculating $\xi$ by the saddle point approximation, one obtains the 
length $L$ of the chain as a derivative of the enthalpy [7]

$$
L=\frac{\partial G}{\partial P}=N\left(r_{\min }-\frac{P}{k}+\frac{3 g P^{2}}{k^{3}}-\frac{3 g k_{B} T}{k^{2}}\right) .
$$

The fact that $L$ scales with $N$ indicates that the expansion is uniform within the chain. Since we are interested in free chains, the pressure $P=0$, and the average expansion per particle is [1, 8]

$$
\langle x\rangle=\frac{L}{N}-r_{\min }=-\frac{3 g k_{B} T}{k^{2}} .
$$

This equation confirms that the expansion of a solid as a function of temperature requires anharmonic terms such as the cubic term proportional to $g$. It is valid at the lowest (linear) order in $g$ or in $T$. At the same order of perturbation, $\left\langle(x-\langle x\rangle)^{2}\right\rangle \simeq k_{B} T / k$, which is the same expression as that obtained for an harmonic potential using the equipartition theorem.

In the specific case of our experimental system of magnetic colloidal chains, the dipolar magnetic part of the interaction potential is attractive and of the form

$$
\beta u_{m}(r)=-\frac{\lambda d^{3}}{r^{3}},
$$

where $\lambda=\beta m^{2} \mu_{0} / 2 \pi d^{3}, m$ is the dipole strength and $\mu_{0}$ is the permeability of vacuum [9, 10]. In experiments, $\lambda$ is in the range of 10-1000. The non-magnetic part of the pair potential is repulsive and can be described as the sum of an hard sphere potential $u_{H S}(r)$ (which is zero for $r>d$ and infinite for $r=d$ ), and a short-ranged electrostatic potential $u_{e l}(r)$. In this paper, we are only interested in the regime where the Debye length $\kappa^{-1}$ is much smaller than the particle size. In this case

$$
u_{e l}(r)=\pi \epsilon \Psi_{0}^{2} d \ln \left(1+e^{-\kappa(r-d)}\right),
$$

in terms of the zeta potential $\Psi_{0}$, which is assumed to be uniform and sufficiently low for the Debye theory to hold [11]. The total pair potential $u(r)$ is

$$
u(r)=u_{H S}(r)+u_{e l}(r)+u_{m}(r) .
$$

The function $u(r)$ is shown as a function of $r$ on figure 1 for different values of $\lambda$. An important feature of this potential is that it is asymmetric for low values of $\lambda$.

We have carried out Monte-Carlo simulations of single chains, which give direct access to the thermal average $\langle r\rangle$. Each chain contains 10 to 20 particles interacting with the potential $u(r)$. We have compared results of such simulations to the low temperature expansion of Eq. 4. As expected, the low temperature approximation correctly describes the simulations with the exact potential in the limit where the effective temperature $1 / \lambda$ is low. This is shown in Fig. 2 for a particular choice of the ionic strength and zeta potential. In the range of magnetic field considered and for these conditions, the low temperature expansion leads to a linear

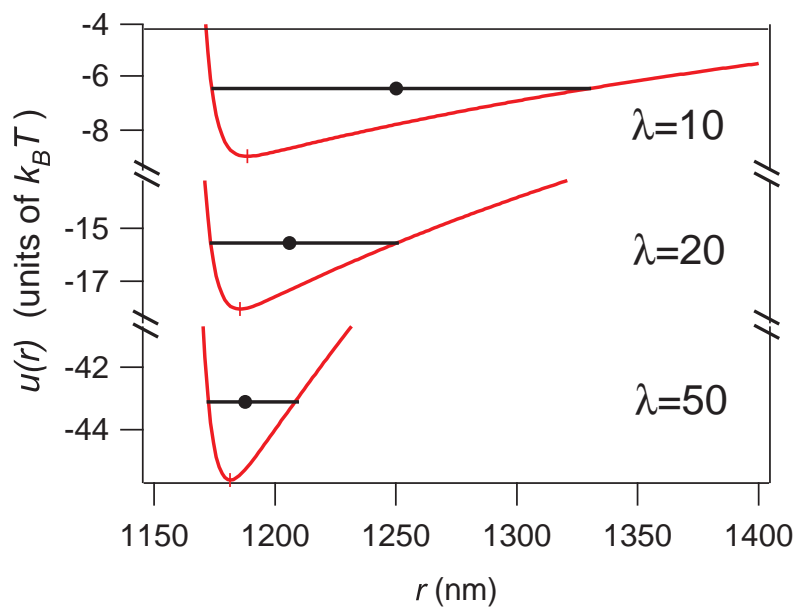

FIG. 1: Total interaction potential $u(r)$ in units of $k_{B} T$ as function of the distance $r$ between the centers of two neighboring colloidal particles. The curves correspond to different values of $\lambda=10,20$ or 50 as shown. The black dot represents the average position $\langle r\rangle$, which is different from the minimum of the potential shown as a vertical stroke. The particle diameter is $d=1150 \mathrm{~nm}$, the zeta potential is $\Psi_{0}=-35 \mathrm{mV}$, and the ionic strength is $5 \mathrm{mM}$, corresponding to a Debye length of about $4 \mathrm{~nm}$.

dependance of $\langle x\rangle$ as function of the effective temperature with $\langle x\rangle \simeq 224.9 / \lambda[\mathrm{nm}]$. Note that error bars on the simulation data of figures 2 and 3 were determined by the method of Ref. [12], they are very small everywhere except for the smallest values of $\lambda$ near the limit of stability of the chains.

From a practical point of view, an attractive feature of this experiment is that it is possible to use it to measure a force acting between neighboring particles. More precisely, this can be done by defining the force $f(\langle r\rangle)$, from the magnetic part of the potential as

$$
f(\langle r\rangle)=\left.\frac{d u_{m}(r)}{d r}\right|_{r=\langle r\rangle}=\frac{3 \lambda k_{B} T d^{3}}{\langle r\rangle^{4}},
$$

where $\langle r\rangle$ is evaluated at the middle of the chain.

This choice of the middle of the chain is made to minimize finite size effects, which we found from our numerical study have only a small effect on the evaluation of forces. We have also found that forces calculated assuming nearest neighbor interactions differ from the case where all neighbors are included [13], only at high magnetic field. In other words, taking into account long range dipolar interactions does not change significantly the force versus distance curves and thus is not essential for analyzing the thermal expansion effect, as we have checked numerically. Furthermore, by comparing the energy of the finite chain to that of the infinite chain, we have found that the force versus distance curves depend only weakly on the chain length, thus confirming the Zhang and Widom model [14], on which Ref. [6] is based. 


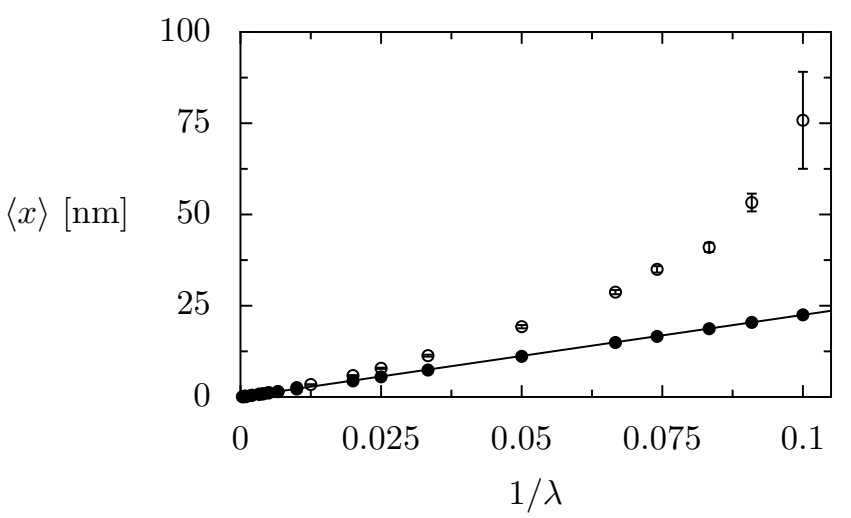

FIG. 2: Average expansion $\langle x\rangle$ versus effective temperature $1 / \lambda$, the empty circles are the simulation data points with the full potential and the filled circles corresponds to the approximation of Eq. 4 with a cubic potential. The particle diameter, zeta potential and ionic strength are the same as in Fig. 1 The solid line is the curve $\langle x\rangle=224.9 / \lambda$ which fits well the points described by Eq. 4 in this range of magnetic field.

The force $f(\langle r\rangle)=f(r)$ defined in this way is equal to the derivative of the electrostatic part of the potential $f_{e l}(r)=-d u_{e l}(r) / d r$ only when $1 / \lambda=0$, i.e. when $\langle x\rangle=0$. Thus a deviation of $f(r)$ away from $f_{e l}(r)$ can be attributed to thermal fluctuations.

As can be seen in fig. 11 at low values of $\lambda$, the potential $u(r)$ is very steep for $r<r_{\min }$ where it can be approximated by its short-ranged part $u_{e l}(r)+u_{H S}(r)$, while $u(r) \simeq u_{m}(r)$ for $r>r_{m i n}$. The most asymmetric situation corresponds therefore to the limit where the Debye length goes to zero. In this case, the magnetic part of the potential is only balanced by the hard sphere repulsion of the colloids. This case is more simple than the previous case because now the position of the minimum of the potential $r_{\min }$ is always $d$ irrespective of $\lambda$. An expansion of the potential about this minimum at $r=d$ leads to

$$
v(x)=p x+\frac{1}{2} k x^{2},
$$

with $p=u_{m}^{\prime}(d)=3 \lambda k_{B} T / d$ and $k=u_{m}^{\prime \prime}(d)=$ $-12 \lambda k_{B} T / d^{2}$. Now by treating the term $k x^{2} / 2$ as a perturbation with respect to the first term $p x$, one obtains to lowest order in $T:\langle x\rangle \simeq k_{B} T / p$, and thus

$$
\langle r\rangle \simeq d\left(1+\frac{1}{3 \lambda}\right) .
$$

This approximation holds for $\lambda \gg 2 / 3$, so essentially for all values of the magnetic field considered here. At the same order in perturbation, $\left\langle(x-\langle x\rangle)^{2}\right\rangle \simeq\left(k_{B} T / p\right)^{2}$, which means that in this case the RMS fluctuations of $x$ are of the same order than their average. For hard-sphere potentials, Eq. 10 can be inverted to obtain $\lambda(\langle r\rangle)$ which leads to the following simple expression for the force

$$
f(\langle r\rangle)=\left(\frac{d}{\langle r\rangle}\right)^{4} \frac{k_{B} T}{\langle r\rangle-d} .
$$

In the limit $T=0$, we recover that $f(\langle r\rangle) \rightarrow$ $3 \mu_{0} m^{2} / 2 \pi d^{4}$. The force $f(\langle r\rangle)$ shown in Fig. 3, has a vertical part near $\langle r\rangle=d$ and a slower power-law decay at larger distances. The vertical part of the curve typical of hard sphere behavior has been observed in Ref. 15] but the slower decay at larger distances has not been analyzed there although it is expected to be present in these experiments.

Let us discuss measurements of the force versus distance [5]. We use Dynal-Invitrogen magnetic beads of diameter $d \simeq 1150 \mathrm{~nm}$, as observed by Dynamic Light Scattering. The polydispersity in size is estimated to be in the range of $1 \%$. We vary the interaction potential by changing the ionic strength, and we measure the mean particle distance using digital video-microscopy [16]. The mean distance is calculated by averaging the distance between particles within the chain and over the time of the experiment. The force versus the mean distance between bead surfaces is shown in Fig. 3. Each set of experiment with a given ionic strength is performed with the same chain in order to reduce errors due polydispersity in particle size. To determine the electrostatic potential, we have fitted the quasi-linear part of the curve for the zeta potential $\Psi_{0}$, and we have found $\Psi_{0} \simeq-35 \mathrm{mV}$. When the Debye length is large, the expansion $\langle x\rangle$ is always negligible in the range of force and distance which can be resolved. As a consequence, we observe only the low temperature regime with a quasi-linear force (in log scale) versus distance profile, characteristic of the electrostatic part of the interaction. For smaller Debye length, thermal fluctuations make the expansion $\langle x\rangle$ observable. A very good agreement is found between the simulations and the experiments except for the smallest Debye length in the region where the deviation from the linear profile occurs. We believe that this discrepancy is not due to a failure of the electrostatic description, which could only happen at much higher values of the surface potential $\Psi_{0}$ than observed here. Instead we attribute the discrepancy to an additional repulsive force, which is due to a polymer layer grafted on the particles [17]. This force is indeed important only when the particles are sufficiently close but irrelevant otherwise. When this additional contribution is taken into account, a good agreement between the experiments and the theory can be obtained for all the ionic strengths studied here.

In Ref. [6], a deviation in the force versus distance measurements (with respect to a linear profile) was reported at high ionic strength and for low field, which we attribute to the thermal expansion effect discussed in this paper. The particles were a factor 5 smaller (there $d=190 \mathrm{~nm}$ ) than in the present study. In fact, for smaller particles, a higher ionic strength is necessary to observe the deviation of Fig. 3 The importance of the particle size or of the Debye length can be determined by evaluating the position of the point where a deviation starts to become observable. If we assume that this happens when $\langle x\rangle \simeq 0.1 \times\langle r\rangle$, using our low temperature approximation, we find that this point corresponds to 


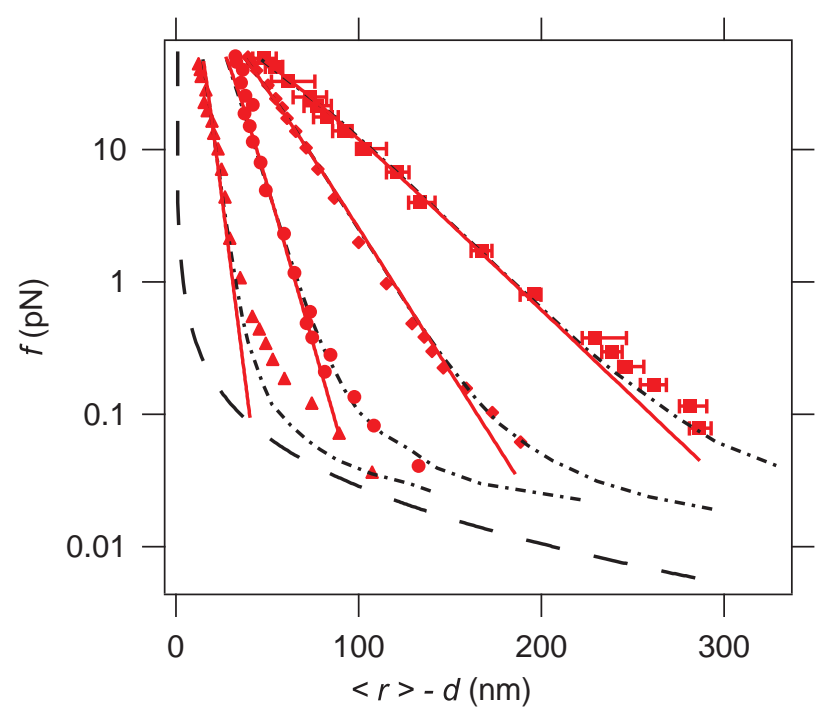

FIG. 3: Force versus the mean distance between the bead surfaces $\langle r\rangle-d$ for different ionic strengths. Symbols correspond to experimental data points, from left to right, $\Delta 5 \mathrm{mM}$, • $1 \mathrm{mM}, 0.25 \mathrm{mM}$ and $0.07 \mathrm{mM}$. The straight lines represent the electrostatic part of the force, the dot-dashed lines represent Monte Carlo simulations, and the dashed line on the left represent the expected force for a hard sphere potential.

( $F=0.7 \mathrm{pN}, h=30 \mathrm{~nm})$ for the curve at $c=5 \mathrm{mM}$ and $(F=0.17 \mathrm{pN}, h=131 \mathrm{~nm})$ for the curve at $c=0.25 \mathrm{mM}$ in agreement with Fig. 3. For lower ionic strength and lower forces, the effect is too weak to be observable.

As the strength of the dipolar interaction is reduced, we observe both in the experiments and in the simulations that thermal fluctuations cause chains to fragment. Independent of fragmentation, disordering begins at the ends [18], where the inter-particle distance is larger due to finite size effects. This effect is similar to edge and surface melting in 2D and 3D solids. We have studied numerically the ratio of the standard deviation of the inter-particle distance divided by the average inter- particle distance, $L_{e}=\sqrt{\left\langle x^{2}\right\rangle} /\langle r\rangle$, which is similar to the Lindemann parameter used to detect the approach to melting in 3D systems. At large $\lambda$, the potential is harmonic and $L_{e}$ is small, whereas for small $\lambda$, the potential becomes anharmonic and $L_{e}$ increases. We find that $L_{e}$ raises very steeply as it approaches a value of the order of 0.1 , which corresponds approximatively to melting in $3 \mathrm{D}$ systems. The threshold value on $\lambda$ where this happens depends very much on dimension, it is much lower in 2D as in 1D for instance.

In order to investigate the $2 \mathrm{D}$ case, we have developed a 2D extension of our Monte Carlo simulation, in which the location of the magnetic dipoles of the chain form a 2D polymer. In addition, we have assumed that the orientations of these dipoles remain frozen in the direction of the applied magnetic field. We have found that the force versus distance curves shown in Fig. 3 do not differ in the $2 \mathrm{D}$ case as compared with the $1 \mathrm{D}$ case, in the range of magnetic field considered here. A difference between the predictions of the 2D and 1D model only arise at very small forces and large distances. In the $2 \mathrm{D}$ case, transverse modes of fluctuations of the chain arise, which are soft bending modes [10]. Such soft modes do not significantly alter the mean distance, which is represented in Fig. 3. This explains the robustness of the 1D model. The region where thermal fluctuations are very large requires further modelling, since there the $2 \mathrm{D}$ or $3 \mathrm{D}$ character of the problem is important.

To summarize, we have analyzed the thermal expansion of a chain of magnetic particles. We have shown that this effect is responsible for a deviation with respect to the quasi-linear force profile observed at low effective temperatures. This effect can be described using a simple $1 \mathrm{D}$ model, and is relevant to the determination of force versus distance using magnetic colloids.

We acknowledge fruitful discussions with P. Chaikin, JF. Joanny, R. Dreyfus and F. Krzakala. D. L. also acknowledges support from the Indo-French Center CEFIPRA (grant 3504-2).
[1] E. A. Stern, P. Līvņš, and Z. Zhang, Phys. Rev. B 43, 8850 (1991).

[2] P. M. Chaikin and T. Lubensky, Principles of condensed matter physics (Cambridge University Press, 1995).

[3] M. M. Calbi, M. W. Cole, S. M. Gatica, M. J. Bojan, and G. Stan, Rev. Mod. Phys. 73, 857 (2001).

[4] K. Zahn, R. Lenke, and G. Maret, Phys. Rev. Lett. 82, 2721 (1999).

[5] R. Dreyfus, D. Lacoste, J. Bibette, and J. Baudry, Eur. Phys. J. E in press (2008).

[6] F. Leal Calderon, T. Stora, O. Mondain Monval, P. Poulin, and J. Bibette, Phys. Rev. Lett. 72, 2959 (1994).

[7] P. M. Ma, Statistical Physics (Cambridge University Press, 1995).

[8] D. Keller, D. Swigon, and C. Bustamente, Biophy. J 84,
733 (2003).

[9] D. Lacoste and T. C. Lubensky, Phys. Rev. E 64, 041506 (2001).

[10] R. Toussaint, E. G. Flekkøy, and G. Helgesen, Phys. Rev. E 74, 051405 (2006), ibid Phys. Rev. Lett. 93, 108304 (2004).

[11] R. J. Hunter, Foundations of Colloid Science (Oxford University Press, 2001).

[12] H. Flyvberg and H. G. Petersen, J. Chem. Phys. 91, 461 (1989).

[13] W. R. Toor and T. C. Halsey, Phys. Rev. A 45, 8617 (1992).

[14] H. Zhang and M. Widom, Phys. Rev. E 51, 2099 (1995).

[15] O. Mondain-Monval, F. Leal-Calderon, and J. Bibette, J. Phys. II France 6, 1313 (1996).

[16] J. C. Crocker and D. G. Grier, J. Colloid Interface Sci. 
179, 298 (1996)

[17] O. Mondain-Monval, A. Espert, P. Omarjee, J. Bibette, F. Leal-Calderon, J. Philip, and J.-F. Joanny, Phys. Rev. Lett. 80, 1778 (1998).
[18] J. M. Philipps and J. G. Dash, J. of Stat. Phys. 120, 721 (2005). 